\title{
Prenatal bed rest in developed and developing regions: a systematic review and meta-analysis
}

\author{
Brittany Matenchuk BSc, Rshmi Khurana MD, Chenxi Cai PhD, Normand G. Boulé PhD, \\ Linda Slater MLIS, Margie H. Davenport PhD
}

Abstract

Background: Bed rest is prescribed by most maternity health care professionals for high-risk pregnancy complications, but the impact of bed rest at home and in hospital has not been explored. Our aim was to quantify the influence of bed rest on maternal/fetal health outcomes in developed and developing regions.

Methods: We conducted a systematic review and meta-analysis of randomized controlled trials. We conducted a structured search through MEDLINE, Embase, CINAHL, Web of Science and the Cochrane Library through Mar. 7, 2019. Trials comparing standard care to standard care plus bed rest after 20 weeks' gestation were assessed. Outcomes included infant birth weight, being small for gestational age, gestational age, premature or very premature birth, perinatal death, admission to the neonatal intensive care unit, preterm rupture of membranes, hypertensive disorders of pregnancy, preeclampsia and gestational diabetes mellitus.

Results: We identified 1191 publications, of which 43 were assessed for eligibility. Sixteen publications reporting on 14 unique studies (2608 women, 3328 infants) were included in the analysis. Overall, maternal/newborn outcomes were similar between women on bed rest and those not on bed rest. In subgroup analyses of developed and developing regions, length of gestation was shorter with bed rest (weighted mean difference $-0.77 \mathrm{wk}, 95 \%$ confidence interval $[\mathrm{Cl}]-1.26$ to $-0.27, I^{2}=0 \%$ ), and the risk of a very premature birth was increased (risk ratio $2.07,95 \% \mathrm{Cl} 1.15$ to $3.73, l^{2}=0 \%$ ) in developed countries.

Interpretation: In developed regions, treatment of complicated pregnancies with more than 1 week of bed rest results in worse newborn outcomes. Additional studies are required to determine whether bed rest or hospital admission improves outcomes in developing regions. PROSPERO Trial registration number: CRD42018099237.

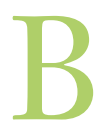
ed rest and activity restriction is prescribed to about $20 \%$ of pregnant women with the intent of improving maternal/fetal health outcomes of high-risk pregnancies complicated by preterm labour, intrauterine growth restriction and hypertensive disorders of pregnancy. ${ }^{1-3}$ Bed rest as a treatment is associated with an economic cost of up to US\$7 billion per year in the United States alone (including hospital admission, lost wages and lost domestic productivity). ${ }^{1,4}$ Previous meta-analyses focused on multiple or singleton pregnancy suggested there is little evidence to support a policy of routine hospital admission for bed rest. ${ }^{5,6}$ However, high heterogeneity was highlighted as an issue for several outcomes. Despite the lack of evidence, bed rest continues to be prescribed by up to $95 \%$ of clinicians. ${ }^{1,7}$ This has resulted in an urgent call for additional research to elucidate the potential benefits (or harms) of bed rest for the woman and her fetus by the World Health Organization and the American College of Obstetricians and Gynecologists. ${ }^{7-9}$

Previous meta-analyses are current to $2017^{5,6,10,11}$ but do not include all available trials. There appears to be a dichotomy between studies on bed rest conducted in developing countries versus developed countries. Bed rest studies conducted in developing countries may be significantly confounded by hospital admission, which may provide patients with increased access to nutritious food and clean water and increased vigilance by medical personnel; this may have substantially less impact on health outcomes in the developed world. However, the influence of the study location's developmental status on the impact of bed rest at home and in hospital has not been explored. Therefore, we conducted a systematic review and meta-analysis of randomized controlled trials (RCTs) that contrasted the effect on maternal/fetal health outcomes of bed rest or activity restriction in conjunction with standard care versus standard care alone (no bed rest) in pregnant women at 20 weeks' gestation or more.

Competing interests: None declared.

This article has been peer reviewed.

Correspondence to: Margie Davenport, margie.davenport@ualberta.ca CMAJ Open 2019. DOI:10.9778/cmajo.20190014 


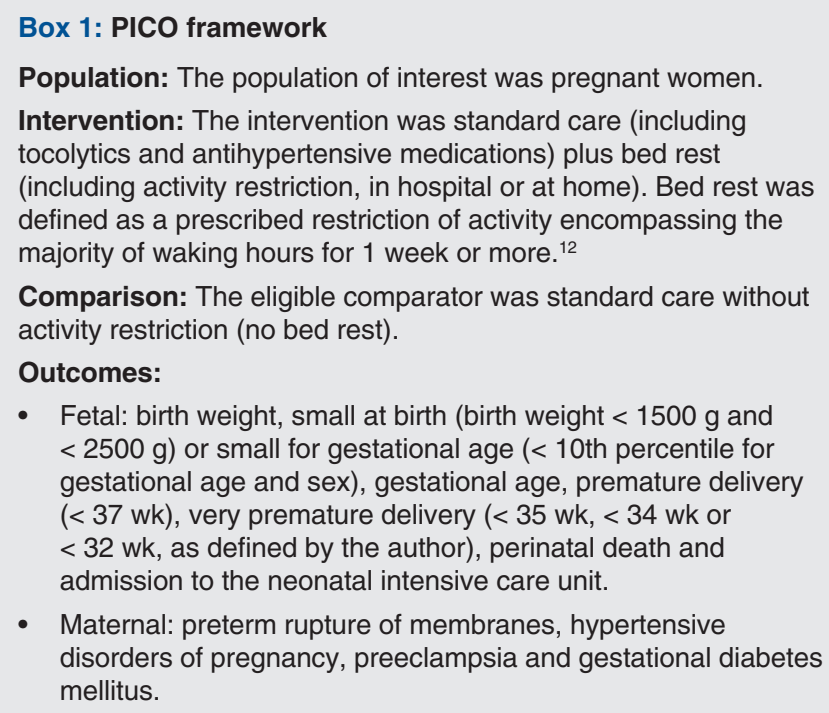

Population: The population of interest was pregnant women. Intervention: The intervention was standard care (including tocolytics and antihypertensive medications) plus bed rest (including activity restriction, in hospital or at home). Bed rest was defined as a prescribed restriction of activity encompassing the majority of waking hours for 1 week or more. ${ }^{12}$

Comparison: The eligible comparator was standard care without activity restriction (no bed rest).

Outcomes:

- Fetal: birth weight, small at birth (birth weight $<1500 \mathrm{~g}$ and $<2500 \mathrm{~g}$ ) or small for gestational age (<10th percentile for gestational age and sex), gestational age, premature delivery ( $<37 \mathrm{wk})$, very premature delivery $(<35 \mathrm{wk},<34 \mathrm{wk}$ or $<32 \mathrm{wk}$, as defined by the author), perinatal death and admission to the neonatal intensive care unit.

- Maternal: preterm rupture of membranes, hypertensive disorders of pregnancy, preeclampsia and gestational diabetes mellitus.

\section{Methods}

\section{Eligibility criteria}

We used the PICO (population, intervention, comparison, outcome[s]) framework to guide this review (Box 1).

\section{Search strategy and study inclusion}

We conducted this systematic review and meta-analysis in accordance with the Cochrane Handbook for Systematic Reviews of Interventions. ${ }^{13} \mathrm{We}$ conducted a structured search through MEDLINE (1946 to Mar. 7, 2019), Embase (1974 to Mar. 7, 2019), CINAHL (1937 to Mar. 7, 2019), Scopus (inception to Mar. 7, 2019), Web of Science (1899 to Mar. 7, 2019) and the Cochrane Library (inception to Mar. 7, 2019). The complete search strategy is given in Appendix 1 (available at www. cmajopen.ca/content/7/3/E435/suppl/DC1). We searched for RCTs investigating the impact of bed rest versus standard care without activity restriction in pregnancy using controlled vocabulary (when available) and text words representing pregnancy/maternal/fetal outcomes or complications combined with terms representing bed rest. We modified the Cochrane RCT filter to exclude the drug therapy floating subheading and to include the term intervention*, and applied it to the searches with the exception of the Cochrane Library. ${ }^{13}$ Studies were not excluded because of language of publication or publication format (e.g., abstracts only). The structured search was created by L.S. and reviewed by a second librarian with systematic review experience. Records identified by the search strategy were independently assessed in duplicate for inclusion by B.M. or C.C., and M.H.D., with N.G.B. acting as arbitrator in the event of disagreement.

\section{Data extraction}

Studies were extracted independently and in duplicate by 2 researchers (B.M. and M.H.D.) using a standardized data collection form including indication for bed rest, duration of bed rest, location of bed rest (e.g., hospital v. home), and any cointerventions used, as well as fetal outcomes of interest (birth weight, being small at birth [birth weight $<1500 \mathrm{~g}$ and $<2500 \mathrm{~g}$ ] or small for gestational age [ $<10$ th percentile for gestational age and sex], gestational age, premature delivery $[<37 \mathrm{wk}]$, very premature delivery $[<35 \mathrm{wk},<34 \mathrm{wk}$ or $<32 \mathrm{wk}$, as defined by the author], perinatal death and admission to the neonatal intensive care unit) and maternal outcomes of interest (preterm rupture of membranes, hypertensive disorders of pregnancy, preeclampsia and gestational diabetes mellitus). When multiple publications from the same trial were identified, data were extracted from all available articles.

\section{Quality measures and risk of bias}

The risk of bias in RCTs was assessed independently and in duplicate by C.C. and M.H.D. following the Cochrane Handbook for Systematic Reviews of Interventions. ${ }^{13}$ All studies were screened for potential sources of bias including selection bias, reporting bias, performance bias, detection bias, attrition bias and "other" sources of bias. The risk of bias across studies was rated as "serious" when studies having the greatest influence on the pooled result (assessed by means of weight [percent] given in forest plots) presented "high" risk of bias. ${ }^{14}$ The quality of the evidence was assessed by C.C. and M.H.D. using the Grading of Recommendations Assessment, Development and Evaluation (GRADE) system. ${ }^{14}$ Evidence from RCTs was rated as high quality by default and then downgraded based on prespecified criteria, including study limitations (weight of studies showed serious risk of bias), inconsistency (heterogeneity was high $\left[I^{2} \geq 50 \%\right]$ or only 1 study was assessed), indirectness (bed-rest-only interventions and bed rest plus cointerventions were combined for analysis), imprecision (95\% confidence interval $[\mathrm{CI}]$ crossed the line of no effect and was wide) and publication bias (substantial evidence of small-study effects).

\section{Statistical analysis}

We conducted statistical analyses using Review Manager v5.2. (Cochrane Collaboration). For continuous outcomes, we examined mean differences between bed-rest and no-bed-rest groups. For binary outcomes, we calculated risk ratios (RRs). We applied inverse-variance weighting to obtain pooled weighted mean differences (WMDs) and RRs using a random effect model. We performed a sensitivity analysis to evaluate whether the effects were different when examining relations between the different indications for bed rest and maternal/ infant outcomes. We conducted the following subgroup analyses, determined a priori: 1) developmental status of the region in the year the study took place based on the World Bank country definition ${ }^{15}$ and 2) single- versus multiple-gestation pregnancies. We used $\chi^{2}$ tests to estimate heterogeneity between trials. The percent of total variability attributable to heterogeneity (i.e., not due to chance) was expressed as the $I$. We explored the source of heterogeneity when intersubgroup heterogeneity was significant $(p<0.05)$. We estimated missing standard deviations (SDs) for outcomes from reported $p$ values and sample sizes, ${ }^{16}$ according to procedures in the Cochrane Handbook for Systematic Reviews of Interventions (section 7.7.3.3). ${ }^{13}$ We used 
GRADEpro GDT (McMaster University and Evidence Prime) to evaluate and tabulate quality of evidence and strength of recommendations. ${ }^{17}$

\section{Ethics approval}

Ethics approval was not required for this systematic review and meta-analysis.

\section{Results}

\section{Study selection}

The literature search identified 1191 potentially relevant studies, 43 of which were assessed for eligibility. Sixteen articles from 14 individual RCTs met our inclusion criteria and were included in the review (Figure 1).

\section{Study characteristics}

The 14 studies assessed in our analysis included 2608 pregnancies (3328 newborns). Nine studies were from developed regions, and 5 were from a developing region (Zimbabwe ${ }^{18-22}$ ). Indications for bed rest in the Zimbabwe studies included multiple-gestation pregnancy ${ }^{18-21}$ and hypertensive disorders of pregnancy.22 Studies evaluating pregnancies in developed regions examined multiple-gestation pregnancy, ${ }^{23-25}$ hypertensive disorders of pregnancy, ${ }^{26,27}$ preterm labour, ${ }^{28,29}$ suspected intrauterine growth restriction, ${ }^{30}$ high risk of preterm birth ${ }^{28}$ and preterm rupture of membranes. ${ }^{31}$

The length of the prescribed bed rest ranged from 1.0 to 9.7 weeks. ${ }^{18-22,25,27,29,31}$ A summary of study characteristics is provided in Table 1.

\section{Quality of evidence}

Overall, the quality of evidence ranged from low to high (Supplementary Table S1, Appendix 2, available at www. cmajopen.ca/content/7/3/E435/suppl/DC1). The most common reasons for downgrading the quality of evidence were serious risk of bias and serious imprecision of the interventions. Common sources of bias included selection bias owing to inadequate generation of a randomized sequence and reporting bias owing to selective outcome reporting.

\section{Synthesis of results}

\section{Fetal outcomes}

Bed rest did not decrease the risk of perinatal death overall (12 RCTs, 1995 births: 782 in developed regions and 1213 in Zimbabwe; RR 1.09, 95\% CI 0.52 to $2.28, I^{2}=37 \%$ (Figure 2) or when separated by developmental status of the country $(p=$ 0.3 for subgroup differences). ${ }^{18-26,29-31}$

Preterm birth $(<37 \mathrm{wk})$ and very preterm birth are the leading causes of perinatal morbidity and mortality. ${ }^{32}$ In our meta-analysis, "very premature" was defined as less than 32 weeks in 2 studies, ${ }^{21,25}$ less than 34 weeks in 4 studies ${ }^{18-20,23}$ and less than 35 weeks in 2 studies..$^{26,29}$ Our analysis of premature birth included 2511 women. ${ }^{18-29,31}$ No difference was found in rates of premature birth between women on bed rest and those not on bed rest (RR 0.98, 95\% CI 0.91 to 1.06 ,

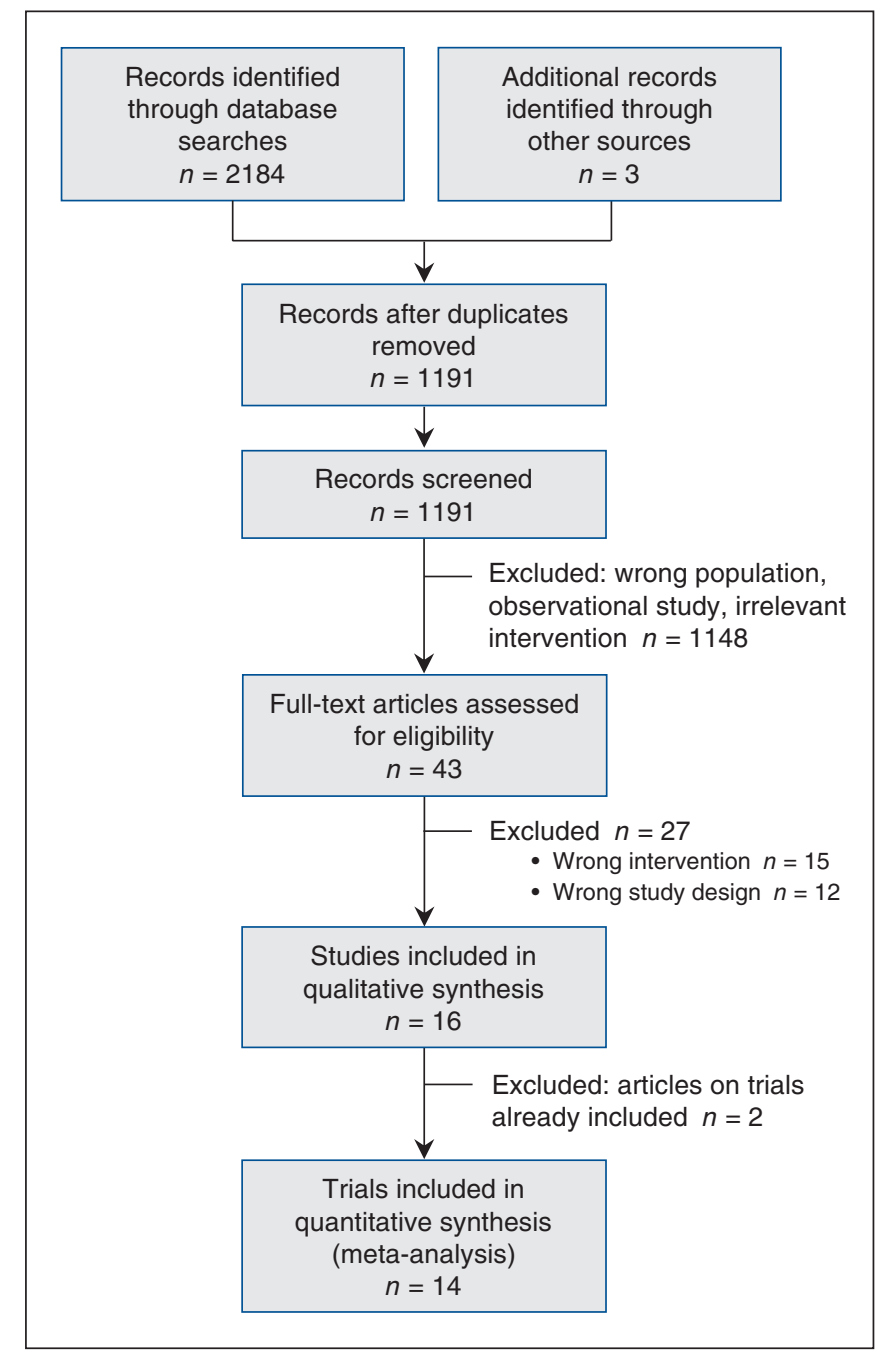

Figure 1: Flow diagram showing study selection.

$I^{2}=0 \%$; low evidence), and subgroup analyses were not significant (Supplementary Figure S1, Appendix 2). High heterogeneity was interrogated in the meta-analysis of very premature birth owing to statistically significant heterogeneity in the developed regions subgroup, which led to the removal of 1 study with a $100 \%$ event rate in both study arms. ${ }^{31}$ Bed rest doubled the risk of having a very premature baby in developed regions (prevalence $6.2 \%$ v. 12.8\%) (RR 2.07, $95 \%$ CI 1.15 to $3.73, I^{2}=0 \%$; moderate evidence) (Figure 3 ) but not the developing region. Subgroup differences were significant $(p=0.03)$.

Subgroup differences were significant for birth weight $(p=$ $0.02)$ and gestational age $(p=0.01)$. Overall, there was highquality evidence that bed rest was not associated with a greater birth weight compared to no bed rest (1492 births; WMD $40 \mathrm{~g}, 95 \% \mathrm{CI}-30 \mathrm{~g}$ to $110 \mathrm{~g}, I^{2}=31 \%$ ) (Supplementary Figure S2, Appendix 2). ${ }^{18-20,22,23,25-27,29-31}$ However, subgroup analysis identified that bed rest modestly increased birth weight in Zimbabwe (WMD $100 \mathrm{~g}$, 95\% CI $40 \mathrm{~g}$ to $170 \mathrm{~g}, I^{2}=$ $0 \% ; p=0.002$ for subgroup differences; high evidence) but had no impact on birth weight in developed regions. 
Table 1 (part 1 of 4): Study characteristics

\begin{tabular}{|c|c|c|c|c|}
\hline Investigator & Country & Participants and methods & Intervention & Outcomes extracted \\
\hline \multicolumn{5}{|c|}{ Developing country } \\
\hline $\begin{array}{l}\text { Crowther et al., }{ }^{20} \\
1989\end{array}$ & Zimbabwe & $\begin{array}{l}139 \text { women with twin pregnancies at } \\
<34 \text { weeks' gestation with cervical } \\
\text { score } \geq-2 \\
\text { Randomization: block randomization } \\
\text { Allocation concealment: } \\
\text { consecutively numbered opaque, } \\
\text { sealed envelopes } \\
\text { Loss to follow-up: } 2 / 70 \text { women in } \\
\text { experimental group were } \\
\text { noncompliant } \\
\text { Exclusion criteria: uncertain } \\
\text { gestational age, cervical suture in } \\
\text { place, antepartum hemorrhage, } \\
\text { hypertension, previous cesarean } \\
\text { delivery and in labour } \\
\text { Recruitment dates: } 1984 \text { onward }\end{array}$ & $\begin{array}{l}\text { Experimental group: in-hospital bed } \\
\text { rest } \\
\text { Mean gestational age at study start } \\
33.3 \text { (SD 1.8) wk } \\
\text { Length: } 2.5 \mathrm{wk} \\
n=70 \\
\text { Control group: conventional } \\
\text { outpatient management; admitted to } \\
\text { hospital if pregnancy complications } \\
\text { occurred } \\
\text { Mean gestational age at study start } \\
33.5 \text { (SD 1.8) wk } \\
\text { Length: } 2.3 \mathrm{wk} \\
n=69\end{array}$ & $\begin{array}{l}\text { Infant: perinatal death, } \\
\text { preterm birth }(<37 \mathrm{wk}), \\
\text { very preterm birth, birth } \\
\text { weight, gestational age, } \\
\text { birth weight }<2500 \mathrm{~g} \text {, birth } \\
\text { weight }<1500 \mathrm{~g}, \mathrm{SGA}, \\
\text { admission to NICU } \\
\text { Maternal: cesarean } \\
\text { delivery, pregnancy-induced } \\
\text { hypertension, PROM }\end{array}$ \\
\hline $\begin{array}{l}\text { Crowther et al., }{ }^{19} \\
1990\end{array}$ & Zimbabwe & $\begin{array}{l}118 \text { women with uncomplicated twin } \\
\text { pregnancies between } 28 \text { and } \\
30 \text { weeks' gestation } \\
\text { Randomization: block randomization } \\
\text { Allocation concealment: numbered } \\
\text { opaque, sealed envelopes } \\
\text { Exclusion criteria: cervical suture, } \\
\text { hypertension, cesarean delivery } \\
\text { scar, antepartum hemorrhage or } \\
\text { uncertain gestational age } \\
\text { 15/58 in experimental group were } \\
\text { noncompliant } \\
\text { Recruitment dates: } 1984-1986\end{array}$ & $\begin{array}{l}\text { Experimental group: in-hospital bed } \\
\text { rest; participants were encouraged to } \\
\text { rest in bed as much as possible, } \\
\text { although voluntary ambulation was } \\
\text { allowed } \\
\text { Mean gestational age at study start } \\
29.1 \text { (SD 1.2) wk } \\
\text { Length: } 7.0 \mathrm{wk} \\
n=58 \\
\text { Control group: advised to continue } \\
\text { normal activities at home; admitted to } \\
\text { hospital if pregnancy complications } \\
\text { occurred } \\
\text { Mean gestational age at study start } \\
29.2 \text { (SD 1.7) wk } \\
\text { Length: } 6.7 \mathrm{wk} \\
n=60\end{array}$ & $\begin{array}{l}\text { Infant: perinatal death, } \\
\text { preterm birth }(<37 \mathrm{wk}), \\
\text { very preterm birth, birth } \\
\text { weight, gestational age, } \\
\text { birth weight }<2500 \mathrm{~g} \text {, birth } \\
\text { weight }<1500 \mathrm{~g}, \mathrm{SGA}, \\
\text { admission to NICU } \\
\text { Maternal: cesarean } \\
\text { delivery, pregnancy-induced } \\
\text { hypertension, PROM }\end{array}$ \\
\hline $\begin{array}{l}\text { Crowther et al., }{ }^{18} \\
1991\end{array}$ & Zimbabwe & $\begin{array}{l}\text { Multiple-gestation births } \\
\text { Randomization: block randomization } \\
\text { Allocation concealment: opaque, } \\
\text { sealed envelopes } \\
\text { Loss to follow-up: none }\end{array}$ & $\begin{array}{l}\text { Experimental group: in-hospital bed } \\
\text { rest } \\
\text { Mean gestational age at study start } \\
29.0 \text { (SD } 4.7) \text { wk } \\
\text { Length: } 5.4 \text { wk } \\
n=10 \\
\text { Control group: advised to continue } \\
\text { normal activities at home; admitted to } \\
\text { hospital if pregnancy complications } \\
\text { occurred } \\
\text { Mean gestational age at study start } \\
29.4 \text { (SD } 3.0 \text { ) wk } \\
\text { Length: } 4.3 \text { wk } \\
n=9\end{array}$ & $\begin{array}{l}\text { Infant: perinatal death, } \\
\text { preterm birth }(<37 \mathrm{wk}), \\
\text { very preterm birth, birth } \\
\text { weight, gestational age, } \\
\text { birth weight }<2500 \mathrm{~g} \text {, birth } \\
\text { weight }<1500 \mathrm{~g}, \mathrm{SGA} \text {, } \\
\text { admission to NICU } \\
\text { Maternal: cesarean } \\
\text { delivery, pregnancy-induced } \\
\text { hypertension, PROM }\end{array}$ \\
\hline $\begin{array}{l}\text { Crowther et al., } 22 \\
1992\end{array}$ & Zimbabwe & $\begin{array}{l}218 \text { women with singleton } \\
\text { pregnancies at } 28-38 \text { weeks' } \\
\text { gestation with nonproteinuric } \\
\text { hypertension (blood pressure } \\
>140 / 90 \mathrm{~mm} \mathrm{Hg} \text { ) } \\
\text { Randomization: block randomization, } \\
\text { stratified } \\
\text { Allocation concealment: opaque, } \\
\text { sealed envelopes } \\
\text { Loss to follow-up: none } \\
\text { Recruitment dates: } 1985-1986\end{array}$ & $\begin{array}{l}\text { Experimental group: admission to } \\
\text { hospital for rest } \\
\text { Mean gestational age at study start } \\
35.3 \text { (SD } 2.6 \text { ) wk } \\
\text { Length: } 3.0 \mathrm{wk} \\
n=110 \\
\text { Control group: normal activity at } \\
\text { home } \\
\text { Mean gestational age at study start } \\
34.6 \text { (SD } 3.0 \text { ) wk } \\
\text { Length: } 3.6 \mathrm{wk} \\
n=108\end{array}$ & $\begin{array}{l}\text { Infant: perinatal death, } \\
\text { preterm birth }(<37 \mathrm{wk}) \text {, } \\
\text { very preterm birth, birth } \\
\text { weight, gestational age, } \\
\text { birth weight }<2500 \mathrm{~g}, \mathrm{SGA} \text {, } \\
\text { admission to NICU } \\
\text { Maternal: cesarean } \\
\text { delivery, preeclampsia }\end{array}$ \\
\hline
\end{tabular}


Table 1 (part 2 of 4): Study characteristics

\begin{tabular}{|c|c|c|c|c|}
\hline Investigator & Country & Participants and methods & Intervention & Outcomes extracted \\
\hline $\begin{array}{l}\text { Saunders } \\
\text { et al., }{ }^{21} 1985\end{array}$ & Zimbabwe & $\begin{array}{l}212 \text { women with twin pregnancies at } \\
\text { about } 30 \text { weeks' gestation } \\
\text { Randomization: randomized; method } \\
\text { not described } \\
\text { Allocation concealment: } \\
\text { consecutively numbered sealed } \\
\text { envelopes } \\
\text { Loss to follow-up: } 11 / 105 \text { in } \\
\text { experimental group declined hospital } \\
\text { admission, and } 2 / 105 \text { delivered } \\
\text { before intervention start; } 1 / 107 \text { in } \\
\text { control group delivered before } \\
\text { intervention start } \\
\text { Recruitment dates: not specified }\end{array}$ & $\begin{array}{l}\text { Experimental group: in-hospital bed } \\
\text { rest from } 32 \text { weeks' gestation until } \\
\text { labour } \\
\text { Mean gestational age at study start } \\
32.7 \mathrm{wk} \\
\text { Length: } 4.6 \mathrm{wk} \\
n=105 \\
\text { Control group: no activity restriction, } \\
\text { at home; admitted to hospital if } \\
\text { pregnancy complications occurred } \\
\text { Gestational age at study start about } \\
32 \text { wk } \\
\text { Length: }>5 \text { wk } \\
n=107\end{array}$ & $\begin{array}{l}\text { Infant: perinatal death, } \\
\text { preterm birth }(<37 \mathrm{wk}), \\
\text { very preterm birth, } \\
\text { gestational age, birth weight } \\
<2500 \mathrm{~g} \text {, birth weight } \\
<1500 \mathrm{~g} \\
\text { Maternal: preeclampsia }\end{array}$ \\
\hline \multicolumn{5}{|c|}{ Developed countries } \\
\hline $\begin{array}{l}\text { Bigelow et al., }{ }^{31} \\
2016\end{array}$ & US & $\begin{array}{l}36 \text { women aged } 18-55 \text { yr with } \\
\text { singleton pregnancies at < } 34 \text { weeks' } \\
\text { gestation with PPROM } \\
\text { Randomization: computer-generated } \\
\text { randomization scheme } \\
\text { Allocation concealment: sealed } \\
\text { envelopes } \\
\text { Exclusion criteria: actively receiving } \\
\text { magnesium sulfate, footling breech } \\
\text { presentation, or maternal or fetal } \\
\text { indication for immediate delivery } \\
\text { Loss to follow-up: } 1 / 18 \text { withdrew from } \\
\text { control allocation } \\
\text { Recruitment dates: not specified }\end{array}$ & $\begin{array}{l}\text { Experimental group: in-hospital bed } \\
\text { rest; instructed to spend majority of } \\
\text { day in bed in reclined or sleeping } \\
\text { position } \\
\text { Mean gestational age at study start } \\
29.2 \text { (SD } 5.7) \text { wk } \\
\text { Length: } 2.7 \text { wk } \\
n=18 \\
\text { Control group: admitted to hospital; } \\
\text { asked to walk for minimum of } 20 \text { min } \\
3 \text { times daily } \\
\text { Mean gestational age at study start } \\
28.9 \text { (SD } 7.6 \text { ) wk } \\
\text { Length: } 1.6 \text { wk } \\
n=17 \\
\text { Participants in both groups given } \\
\text { latency antibiotics } \pm \text { ampicillin/ } \\
\text { amoxicillin and erythromycin for up to } \\
7 \text { d and } 48-h \text { course of } \\
\text { intramuscularly administered } \\
\text { betamethasone }\end{array}$ & $\begin{array}{l}\text { Infant: perinatal death, } \\
\text { preterm birth }(<37 \mathrm{wk}) \\
\text { very preterm birth, birth } \\
\text { weight, gestational age, } \\
\text { admission to NICU } \\
\text { Maternal: cesarean delivery }\end{array}$ \\
\hline $\begin{array}{l}\text { Dodd et al., }{ }^{23} \\
2005\end{array}$ & Australia & $\begin{array}{l}7 \text { women with triplet pregnancies } \\
\text { Randomization: randomization } \\
\text { schedule used variable blocks with } \\
\text { stratification by parity } \\
\text { Allocation concealment: third party } \\
\text { opened consecutively numbered } \\
\text { opaque, sealed envelopes and } \\
\text { reported allocation over telephone } \\
\text { Loss to follow-up: none } \\
\text { Recruitment dates: } 1996-2003\end{array}$ & $\begin{array}{l}\text { Experimental group: in hospital from } \\
24 \text { to } 30 \text { weeks' gestation; biweekly } \\
\text { assessment; allowed to leave ward } \\
\text { during weekends; encouraged to rest } \\
\text { at home following discharge } \\
\text { Mean gestational age at study start } \\
23.4 \text { (SD 1.7) wk } \\
\text { Length: about } 6 \mathrm{wk} \\
n=3 \\
\text { Control group: advised to continue } \\
\text { normal activity at home; } \\
\text { biweekly in-clinic assessment } \\
\text { Mean gestational age at study start } \\
22.0 \text { (SD 1.8) wk } \\
\text { Length: about } 6 \mathrm{wk} \\
n=4\end{array}$ & $\begin{array}{l}\text { Infant: perinatal death, } \\
\text { preterm birth }(<37 \mathrm{wk}), \\
\text { very preterm birth, birth } \\
\text { weight, gestational age, } \\
\text { birth weight }<2500 \mathrm{~g} \text {, birth } \\
\text { weight }<1500 \mathrm{~g} \\
\text { Maternal: cesarean } \\
\text { delivery, pregnancy-induced } \\
\text { hypertension }\end{array}$ \\
\hline
\end{tabular}

When gestational age at birth was considered as a continuous variable, women who were on bed rest delivered babies at an earlier gestational age in developed regions (WMD $-0.77 \mathrm{wk}, 95 \%$ CI -1.26 to $-0.27, I^{2}=0 \%$; moderate evidence) (Figure 4) but not in the developing region (WMD $-0.04 \mathrm{wk}, 95 \% \mathrm{CI}-0.35$ to $0.26, I^{2}=6 \%$; high-quality evidence)..$^{18-25,29,30,31}$
Overall, moderate-quality evidence indicated that bed rest did not decrease the risk of birth weight less than $2500 \mathrm{~g}$ (1837 births; RR 0.92, 95\% CI 0.85 to $1.00, I^{2}=0 \%$ [Supplementary Figure S3, Appendix 2]). ${ }^{185,27,29}$ Bed rest decreased the risk of delivering a baby weighing less than $2500 \mathrm{~g}$ in Zimbabwe (RR 0.89 , 95\% CI 0.81 to $0.98, I^{2}=0 \%$; highquality evidence) but not in developed regions. 


\begin{tabular}{|c|c|c|c|c|}
\hline Investigator & Country & Participants and methods & Intervention & Outcomes extracted \\
\hline $\begin{array}{l}\text { Elliott et al., }{ }^{29} \\
2005\end{array}$ & US & $\begin{array}{l}73 \text { women with singleton } \\
\text { pregnancies experiencing preterm } \\
\text { labour with negative fetal fibronectin } \\
\text { recruited from } 4 \text { tertiary hospitals in } \\
\text { southwestern United States } \\
\text { Randomization: computer-generated } \\
\text { randomization schedule } \\
\text { Allocation concealment: reported to } \\
\text { study coordinator by third party, who } \\
\text { opened opaque, sealed envelopes } \\
\text { Inclusion criteria: }>14 \text { yr of age, } \\
\text { intact membranes, documented } \\
\text { uterine contractions of }>6 / \text { h at } \\
\text { admission, } 23-336 / 7 \text { weeks' } \\
\text { gestation, }<3 \mathrm{~cm} \text { cervical dilatation } \\
\text { Recruitment dates: November } \\
\text { 1997-September } 2000\end{array}$ & $\begin{array}{l}\text { Experimental group: activity } \\
\text { restriction at home; } 2 \text { weekly clinic } \\
\text { visits, followed by biweekly clinic visits } \\
\text { Mean gestational age at study start } \\
30.7 \text { (SD } 2.7 \text { ) wk } \\
\text { Length: } 5.9 \text { wk } \\
n=36 \\
\text { Control group: instructed to resume } \\
\text { normal activities, including work } \\
\text { responsibilities, at home; } 2 \text { weekly } \\
\text { clinic visits followed by biweekly clinic } \\
\text { visits } \\
\text { Mean gestational age at study start } \\
31.0 \text { (SD } 6.3 \text { ) wk } \\
\text { Length: } 6.6 \text { wk } \\
n=37\end{array}$ & $\begin{array}{l}\text { Infant: perinatal death, } \\
\text { preterm birth }(<37 \mathrm{wk}) \text {, } \\
\text { very preterm birth, birth } \\
\text { weight, gestational age, } \\
\text { birth weight }<2500 \mathrm{~g} \text {, birth } \\
\text { weight }<1500 \mathrm{~g} \text {, admission } \\
\text { to NICU } \\
\text { Maternal: none }\end{array}$ \\
\hline $\begin{array}{l}\text { Hartikainen- } \\
\text { Sorri et al., }{ }^{24} \\
1984\end{array}$ & Finland & $\begin{array}{l}73 \text { women with twin pregnancies } \\
\text { Randomization: based on year of } \\
\text { birth } \\
\text { Allocation concealment: not } \\
\text { mentioned } \\
\text { Loss to follow-up: } 5 \text { women excluded } \\
\text { from experimental group owing to } \\
\text { program refusal } \\
\text { Recruitment dates: } 1979-1980\end{array}$ & $\begin{array}{l}\text { Experimental group: routine hospital } \\
\text { rest } \\
\text { Gestational age at study start > } 29 \mathrm{wk} \\
\text { Length: until delivery (mean } 36.7 \text { [SD } \\
2.4] \text { wk) } \\
n=28 \\
\text { Control group: specialized } \\
\text { outpatient antenatal care; weekly } \\
\text { clinic visits; admitted to hospital if } \\
\text { complications occurred } \\
\text { Gestational age at study }>29 \mathrm{wk} \\
\text { Length: until delivery (mean } 37.4 \text { [SD } \\
1.8] \text { wk) } \\
n=45\end{array}$ & $\begin{array}{l}\text { Infant: perinatal death, } \\
\text { preterm birth }(<37 \mathrm{wk}), \\
\text { gestational age, birth weight } \\
<2500 \mathrm{~g} \text {, birth weight } \\
<1500 \mathrm{~g} \\
\text { Maternal: pregnancy- } \\
\text { induced hypertension }\end{array}$ \\
\hline $\begin{array}{l}\text { Hobel et al., }{ }^{28} \\
1994\end{array}$ & US & $\begin{array}{l}1774 \text { women at high risk for preterm } \\
\text { birth based on scoring of risk factors } \\
\text { at }<31 \text { weeks' gestation } \\
\text { Women in intervention hospitals } \\
\text { were randomized to } 1 \text { of } \\
5 \text { interventions } \\
\text { Randomization: method not } \\
\text { described } \\
\text { Allocation concealment: not } \\
\text { described } \\
\text { Loss to follow-up: not described }\end{array}$ & $\begin{array}{l}\text { 1. Experimental group: bed rest at } \\
\text { home } \\
\text { 2. Control group 1: placebo } \\
\text { 3. Progestin: women administered } \\
\text { progestin; not included in meta- } \\
\text { analyses } \\
\text { 4. Social support: women given } \\
\text { social support; not included in } \\
\text { meta-analyses } \\
\text { 5. Control group 2: no intervention } \\
\text { Length: < } 31 \text { wk until birth } \\
\text { All participants in intervention } \\
\text { hospitals were given education } \\
\text { intervention consisting of identification } \\
\text { of preterm labour, steps to take if } \\
\text { signs of preterm labour occurred and } \\
\text { prevention strategies }\end{array}$ & $\begin{array}{l}\text { Infant: preterm birth } \\
(<37 \mathrm{wk}) \\
\text { Maternal: none }\end{array}$ \\
\hline $\begin{array}{l}\text { Laurin et al., }{ }^{30} \\
1987\end{array}$ & Sweden & $\begin{array}{l}\text { Women with singleton pregnancies } \\
\text { with estimated weight deviation } \\
\text { (suspected intrauterine growth } \\
\text { restriction) of }>20 \% \text { at } 32 \text { weeks' } \\
\text { gestation or }>15 \% \text { at } 34 \text { weeks' } \\
\text { gestation } \\
\text { Randomization: quasi-random based } \\
\text { on even and odd year of birth } \\
\text { (maternal) } \\
\text { Allocation: not described } \\
\text { Loss to follow-up: } 15 / 49 \text { in } \\
\text { experimental group and } 8 / 58 \text { in } \\
\text { control group did not fulfill } \\
\text { requirements of their allocation } \\
\text { Recruitment dates: } 1982-1983\end{array}$ & $\begin{array}{l}\text { Experimental group: admitted to } \\
\text { hospital; advised to rest in bed } 22 \mathrm{~h} \\
\text { per day; allowed to rest at home on } \\
\text { weekends } \\
\text { Gestational age at study start }<35 \mathrm{wk} \\
\text { Length: > } 3 \text { wk on average } \\
n=34 \\
\text { Control group: normal activity at } \\
\text { home; discontinuation of work duties } \\
\text { Gestational age at study start }<35 \mathrm{wk} \\
\text { Length: }>4 \text { wk on average } \\
n=50\end{array}$ & $\begin{array}{l}\text { Infant: perinatal death, birth } \\
\text { weight, gestational age } \\
\text { Maternal: cesarean delivery }\end{array}$ \\
\hline
\end{tabular}




\begin{tabular}{|c|c|c|c|c|}
\hline Investigator & Country & Participants and methods & Intervention & Outcomes extracted \\
\hline $\begin{array}{l}\text { Leung et al.., }{ }^{27} \\
1998\end{array}$ & $\begin{array}{l}\text { Hong } \\
\text { Kong }\end{array}$ & $\begin{array}{l}88 \text { women with singleton } \\
\text { pregnancies at } 28-38 \text { weeks' } \\
\text { gestation with diastolic blood } \\
\text { pressure } 90-100 \mathrm{~mm} \mathrm{Hg} \\
\text { Randomization: not described } \\
\text { Allocation concealment: } \\
\text { consecutively numbered opaque, } \\
\text { sealed envelopes } \\
\text { Loss to follow-up: infant outcomes } \\
\text { for } 13 \text { pregnancies in experimental } \\
\text { group and } 8 \text { pregnancies in control } \\
\text { group not presented owing to lack of } \\
\text { hypertension } \\
\text { Exclusion criteria: proteinuria or } \\
\text { symptoms of severe preeclampsia } \\
\text { Recruitment dates: May 1995- } \\
\text { November } 1996\end{array}$ & $\begin{array}{l}\text { Experimental group: admitted to } \\
\text { hospital and advised to rest in bed as } \\
\text { much as possible } \\
\text { Mean gestational age at study start } \\
33.2 \text { (SD 2.9) wk } \\
n=44 \\
\text { Control group: normal activity at } \\
\text { home; daily proteinuria testing at } \\
\text { home and weekly clinic visits; } \\
\text { admitted to hospital if proteinuria, } \\
\text { severe preeclampsia or fetal growth } \\
\text { restriction developed } \\
\text { Mean gestational age at study } 33.1 \\
\text { (SD } 3.0 \text { ) wk } \\
n=44\end{array}$ & $\begin{array}{l}\text { Infant: birth weight, birth } \\
\text { weight }<2500 \mathrm{~g}, \mathrm{SGA}, \\
\text { admission to NICU } \\
\text { (reported only for } \\
\text { pregnancies in which } \\
\text { hypertension developed } \\
\text { [31 inpatients and } \\
36 \text { outpatients]) } \\
\text { Maternal: cesarean } \\
\text { delivery, pregnancy-induced } \\
\text { hypertension }\end{array}$ \\
\hline $\begin{array}{l}\text { MacLennan } \\
\text { et al., }{ }^{25} 1990\end{array}$ & Australia & $\begin{array}{l}141 \text { women with multiple-gestation } \\
\text { pregnancies (twins) } \\
\text { Randomization: computer-generated } \\
\text { list of random numbers } \\
\text { Allocation concealment: patient and } \\
\text { research coordinator blinded to } \\
\text { allocation number meaning } \\
\text { Exclusion criteria: hypertension, } \\
\text { polyhydramnios, antepartum } \\
\text { hemorrhage, preterm labour or } \\
\text { rupture of membranes. } \\
\text { Loss to follow-up: } 13 / 69 \text { participants } \\
\text { allocated to experimental group did } \\
\text { not complete study } \\
\text { Recruitment dates: not specified }\end{array}$ & $\begin{array}{l}\text { Experimental group: in hospital from } \\
26 \text { to } 30 \text { weeks' gestation; allowed to } \\
\text { leave on weekends } \\
\text { Mean gestational age at study start } \\
26.0 \text { (SD } 2.1) \mathrm{wk} \\
\text { Length: } 4 \mathrm{wk} \\
n=69 \\
\text { Control group: advised to continue } \\
\text { normal activities at home and visit } \\
\text { clinic every } 2 \mathrm{wk} \\
\text { Mean gestational age at study start } \\
26.0 \text { (SD 2.1) wk } \\
\text { Length: } 4 \mathrm{wk} \\
n=72\end{array}$ & $\begin{array}{l}\text { Infant: perinatal death, } \\
\text { preterm birth }(<37 \mathrm{wk}) \text {, } \\
\text { very preterm birth, birth } \\
\text { weight, gestational age, } \\
\text { birth weight }<2500 \mathrm{~g} \text {, birth } \\
\text { weight }<1500 \mathrm{~g} \text {, admission } \\
\text { to NICU } \\
\text { Maternal: cesarean } \\
\text { delivery, pregnancy-induced } \\
\text { hypertension, PROM, GDM }\end{array}$ \\
\hline Mathews, ${ }^{26} 1977$ & UK & $\begin{array}{l}135 \text { women with singleton } \\
\text { pregnancies complicated by mild } \\
\text { nonalbuminuric and nonsymptomatic } \\
\text { hypertension (diastolic blood } \\
\text { pressure } 90-109 \mathrm{~mm} \mathrm{Hg} \text { ) after } \\
28 \text { weeks' gestation } \\
\text { Randomization: randomized; method } \\
\text { not described } \\
\text { Allocation concealment: previously } \\
\text { prepared cards in envelopes } \\
\text { Loss to follow-up: patients excluded } \\
\text { from trial if they refused hospital } \\
\text { admission }\end{array}$ & $\begin{array}{l}\text { Sedated group } \\
\text { Experimental group: admitted to } \\
\text { hospital and kept in bed aside from } \\
\text { meals and toileting; administered } \\
\text { phenobarbitone, } 15 \mathrm{mg} 3 \text { times daily } \\
\text { Gestational age at study start }>28 \mathrm{wk} \\
\text { Length: } 97.2 \% \text { delivered after } 37 \mathrm{wk} \\
n=36 \\
\text { Control group: advised to resume } \\
\text { normal activity at home; administered } \\
\text { phenobarbitone, } 15 \mathrm{mg} 3 \text { times daily } \\
\text { Gestational age at study start }>28 \mathrm{wk} \\
\text { Length: } 97.2 \% \text { delivered after } 37 \mathrm{wk} \\
n=36 \\
\text { Nonsedated group } \\
\text { Experimental group: admitted to } \\
\text { hospital and kept in bed aside from } \\
\text { meals and toileting } \\
\text { Gestational age at study start } \\
>28 \mathrm{wk} \\
\text { Length: } 97.1 \% \text { delivered after } 37 \mathrm{wk} \\
n=35 \\
\text { Control group: advised to resume } \\
\text { normal activity at home } \\
\text { Gestational age at study start }>28 \mathrm{wk} \\
\text { Length: } 100 \% \text { delivered after } 37 \mathrm{wk} \\
n=28\end{array}$ & $\begin{array}{l}\text { Infant: perinatal death, } \\
\text { preterm birth }(<37 \mathrm{wk}) \text {, } \\
\text { very preterm birth, birth } \\
\text { weight, gestational age, } \\
\text { birth weight }<2500 \mathrm{~g}, \mathrm{SGA} \\
\text { Maternal: cesarean } \\
\text { delivery, pregnancy-induced } \\
\text { hypertension }\end{array}$ \\
\hline
\end{tabular}




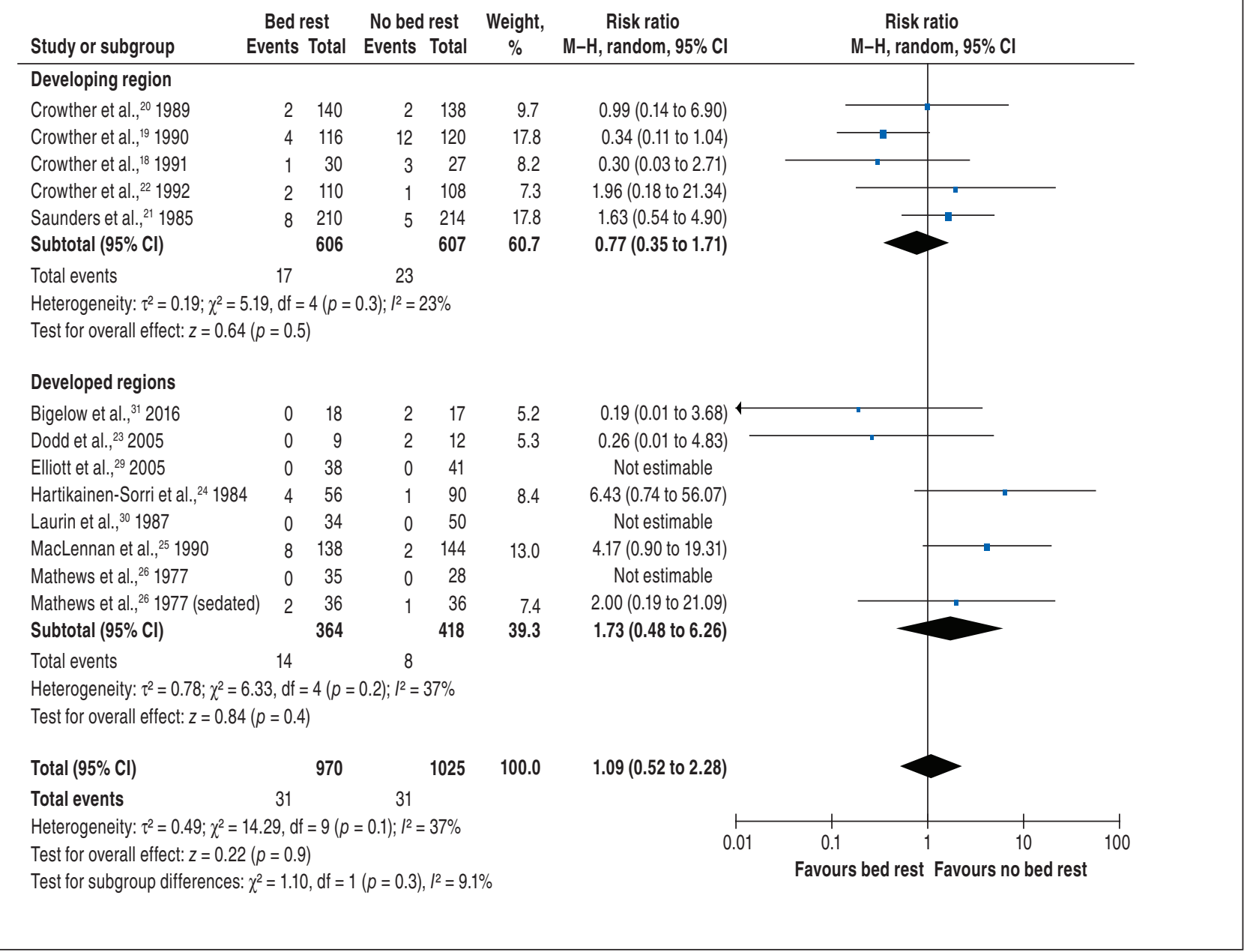

Figure 2: Effect of bed rest (experimental) versus no bed rest (control) on perinatal death. Note: $\mathrm{Cl}=$ confidence interval, $\mathrm{df}=\mathrm{degrees}$ of freedom, $\mathrm{M}-\mathrm{H}=$ Mantel-Haenszel.

The risk of delivering a newborn weighing less than $1500 \mathrm{~g}$ (Supplementary Figure S4, Appendix 2), ${ }^{18-21,23-25,29}$ being small for gestational age (Supplementary Figure S5, Appendix 2) ) $^{18-20,22,26,27}$ or being admitted to the neonatal intensive care unit (Supplementary Figure S6, Appendix 2) ${ }^{18-20,22,25,27,29,31}$ was similar between the bed-rest and no-bed-rest groups. Subgroup analyses were not statistically significant.

\section{Maternal outcomes}

Overall, there was low-quality evidence from 10 RCTs (963 women) regarding the association between bed rest and cesarean birth. ${ }^{18-20,22,23,25-27,30,31}$ The pooled estimate indicated that bed rest did not reduce the rate of cesarean delivery (RR $1.00,95 \%$ CI 0.74 to $1.34, I^{2}=31 \%$ ) (Supplementary Figure S7, Appendix 2). Subgroup analyses were not statistically significant.

Six RCTs (559 women) with low-quality evidence indicated that bed rest did not reduce the risk of hypertensive disorders of pregnancy (RR $0.85,95 \%$ CI 0.51 to $1.42, I^{2}=$ $25 \%$ ) (Supplementary Figure S8, Appendix 2). ${ }^{18-20,23-26}$ Bed rest also did not reduce the rate of preeclampsia (RR 0.98, 95\% CI 0.80 to 1.19) (Supplementary Figure S9, Appendix 2) or preterm rupture of membranes (438 women; RR 1.38, $95 \%$ CI 0.85 to $2.26, I^{2}=0 \%$ ) (Supplementary Figure S10, Appendix 2). ${ }^{18-20,25}$

One moderate-quality RCT ${ }^{25}$ (downgraded owing to serious inconsistency) (141 women) showed that bed rest did not reduce the rate of gestational diabetes (RR 1.04, 95\% CI 0.22 to 4.99) (Supplementary Figure S11, Appendix 2). Funnel plots showed that the outcomes examined were not influenced by publication bias (Supplementary Figures S12 and S13, Appendix 2).

\section{Sensitivity analyses}

Eight of the 14 studies included multiple-gestation pregnancies. $^{18-21,23,25-27}$ When stratified for singleton or multiple gestation, perinatal death, premature birth at less than 37 weeks, gestational age, birth weight less than $1500 \mathrm{~g}$, birth weight less than $2500 \mathrm{~g}$, being small for gestational age, admission to neonatal intensive care unit, cesarean delivery, preterm 




Figure 3: Effect of bed rest (experimental) versus no bed rest (control) on very preterm birth. The study by Bigelow and colleagues ${ }^{31}$ was removed owing to its influence on heterogeneity (heterogeneity of developed regions subgroup $p<0.001 ; 100 \%$ event rate in both study arms). Note: $\mathrm{Cl}=$ confidence interval, $\mathrm{df}=$ degrees of freedom, $\mathrm{M}-\mathrm{H}=$ Mantel-Haenszel.

rupture of membranes, gestational diabetes, pregnancyinduced hypertension and preeclampsia were all similar between the no-bed-rest and bed-rest groups (Supplementary Figures S14-S27, Appendix 2). Although RCTs examining bed rest for multiple births were more common in the developing region than in developed regions, stratification by singleton- versus multiple-gestation birth explained significantly less heterogeneity than stratification by developmental status of the region.

\section{Interpretation}

Overall, maternal and fetal outcomes were similar between women on bed rest and those not on bed rest. However, stratification by the developmental status of the region explained a significant amount of heterogeneity; this has been noted in previous meta-analyses on this topic. ${ }^{5,10,11}$ Stratification identified a divergent impact of bed rest between groups such that bed rest in developed regions decreased gestational age by 5.4 days and increased the risk of delivering a very premature baby. In contrast, bed rest in the developing region increased birth weight by $100 \mathrm{~g}$ and decreased the risk of delivering a baby weighing less than $2500 \mathrm{~g}$.

Six Cochrane reviews have previously examined the impact of bed rest on maternal and fetal outcomes including the prevention of preeclampsia ( 2 studies, $n=106),{ }^{33}$ improving outcomes of pregnancies complicated by hypertension (4 studies, $n=449),{ }^{10}$ the prevention of preterm birth ( 1 study, $\left.n=1266\right),{ }^{3}$ impaired fetal growth (1 study, $n=107)^{34}$ and multiple gestation (7 studies combining complicated and uncomplicated pregnancies, $n=713 ;{ }^{5} 6$ studies including strict or partial bed rest, $n=636) .{ }^{11}$ In all cases, the investigators concluded that there was insufficient evidence for or against the use of bed rest to improve maternal/fetal health outcomes as a result of small samples and high heterogeneity. In nonpregnant populations, the physiological effects of bed rest are not altered by the indication for bed rest; ${ }^{35}$ thus, we combined all indications for bed rest during pregnancy. Stratification by developmental status of the region resulted in subgroup heterogeneity of $40 \%$ or less for all but 1 subgroup analysis (55\%). Hospital admission may explain some of the differences observed between the influence of bed rest in developed versus developing regions. 




Figure 4: Effect of bed rest (experimental) versus no bed rest (control) on gestational age. Note: $\mathrm{Cl}=\mathrm{confidence}$ interval, df $=$ degrees of freedom, IV = inverse variance, SD = standard deviation.

Although bed rest has been shown to decrease maternal weight gain in developed countries, ${ }^{36}$ admission to hospital in developing countries may afford access to proper nutrition, sanitation, clean water and medical professionals. These factors may overcome some of the negative physiological effects of bed rest in selected populations.

It is beyond the scope of this review to determine whether bed rest or hospital admission itself improved birth weight in studies in Zimbabwe. Additional work is required to dissect the influence of hospital admission versus bed rest in developing regions. Bed rest is likely to drive inflammation, which may increase the risk of preterm birth, necessitating further research on the levels of inflammatory cytokines in pregnant women on bed rest. Although there is a reduction in blood pressure during bed rest, ${ }^{37}$ other mechanisms of harm such as endothelial dysfunction may lead to further negative effects in pregnancies complicated by hypertension or preeclampsia. As a result, further research may be required in developed regions on the influence of bed rest on pregnancies complicated by hypertension.

\section{Limitations}

All trials of bed rest in developing regions were conducted in Zimbabwe. As the rates of maternal and fetal morbidity and mortality in Zimbabwe are among the highest in the world, ${ }^{38,39}$ our findings from this country may not be generalizable to other developing regions. Furthermore, as all the Zimbabwe studies were conducted between 1984 and 1992, this may limit the applicability of the results for developing countries, as obstetrical practice will have changed over time. In the Zimbabwe studies, bed rest was conducted in hospital, and no bed rest was conducted at home. In developing countries, low birth weight is associated with hypertensive disorders of pregnancy, preeclampsia, nutritional status of the woman, anemia and access to health care. ${ }^{40}$ Hospital admission may afford improved access to skilled health care workers, sanitation and nutritional status monitoring, thereby reducing the risk of low birth weight. ${ }^{40}$

\section{Conclusion}

Our analyses showed that, in developed regions, 1 additional baby was born very premature for every 15.1 women treated with bed rest. In conjunction with the overwhelming evidence of negative maternal health consequences of prenatal bed rest, our results suggest that bed rest increases the risk of serious negative consequences for newborns in developed regions. In developing regions, bed rest appears to have a minimal positive effect on birth weight, but this finding may be confounded by the effects of hospital admission. 


\section{References}

1. Goldenberg RL, Cliver SP, Bronstein J, et al. Bed rest in pregnancy. Obstet Gynecol 1994;84:131-6.

2. Durrance CP, Guldi M. Maternal bed rest and infant health. Am 7 Health Econ 2015;1:345-73

3. Sciscione AC. Maternal activity restriction and the prevention of preterm birth. Am 7 Obstet Gynecol 2010;202:232. e1-5.

4. Biggio JR Jr. Bed rest in pregnancy: time to put the issue to rest. Obstet Gynecol 2013;121:1158-60.

5. Crowther CA, Han SJ. Hospitalisation and bed rest for multiple pregnancy. Cochrane Database Syst Rev 2010;(7):CD000110.

6. Sosa CG, Althabe F, Belizan JM, et al. Bed rest in singleton pregnancies for preventing preterm birth. Cochrane Database Syst Rev 2015;(3):CD003581.

7. Fox NS, Gelber SE, Kalish RB, et al. The recommendation for bed rest in the setting of arrested preterm labor and premature rupture of membranes. Am $\mathcal{F}$ Obstet Gynecol 2009;200:165 e1-6.

8. McCall CA, Grimes DA, Lyerly AD. "Therapeutic" bed rest in pregnancy: unethical and unsupported by data. Obstet Gynecol 2013;121:1305-8.

9. WHO recommendations for prevention and treatment of pre-eclampsia and eclampsia. Geneva: World Health Organization; 2011.

10. Meher S, Abalos E, Carroli G. Bed rest with or without hospitalisation for hypertension during pregnancy. Cochrane Database Syst Rev 2005;(4):CD003514.

11. da Silva Lopes K, Takemoto Y, Ota E, et al. Bed rest with and without hospitalisation in multiple pregnancy for improving perinatal outcomes. Cochrane Database Syst Rev 2017;(3):CD012031.

12. Maloni JA. Lack of evidence for prescription of antepartum bed rest. Expert Rev Obstet Gynecol 2011;6:385-93.

13. Higgins JPT, Green S, editors. Cochrane handbook for systematic reviews of interventions. Version 5.1.0 [updated March 2011]. Oxford (UK): Cochrane Collaboration; 2011.

14. Schünemann HBJ, Brożek J, Guyatt G, et al., editors. GRADE bandbook for grading quality of evidence and strength of recommendations. GRADE Working Group; [updated October 2013]. Available: guidelinedevelopment.org/handbook (accessed 2019 June 5).

15. World Bank Country and Lending Groups. Washington: World Bank; 2018. Available: http://data.worldbank.org/about/country-classifications (accessed 2019 Jan. 15).

16. Jadad AR, Moore RA, Carroll D, et al. Assessing the quality of reports of randomized clinical trials: Is blinding necessary? Control Clin Trials 1996;17:1-12.

17. GRADEpro GDT: GRADEpro Guideline Development Tool [software]. Hamilton (ON): McMaster University [developed by Evidence Prime]; 2015. Available: gradepro.org (accessed 2019 June 5).

18. Crowther CA, Verkuyl DA, Ashworth MF, et al. The effects of hospitalization for bed rest on duration of gestation, fetal growth and neonatal morbidity in triplet pregnancy. Acta Genet Med Gemellol (Roma) 1991;40:63-8.

19. Crowther CA, Verkuyl DA, Neilson JP, et al. The effects of hospitalization for rest on fetal growth, neonatal morbidity and length of gestation in twin pregnancy. Br 7 Obstet Gynaecol 1990;97:872-7.

20. Crowther CA, Neilson JP, Verkuyl DA, et al. Preterm labour in twin pregnancies: Can it be prevented by hospital admission? Br 7 Obstet Gynaecol 1989; 96:850-3.

21. Saunders MC, Dick JS, Brown IM, et al. The effects of hospital admission for bed rest on the duration of twin pregnancy: a randomised trial. Lancet 1985;2: 793-5.

22. Crowther C, Bouwmeester A, Ashurst HM. Does admission to hospital for bed rest prevent disease progression or improve fetal outcome in pregnancy complicated by non-proteinuric hypertension? Br 7 Obstet Gynaecol 1992;99:13-7.

23. Dodd JM, Crowther CA. Hospitalisation for bed rest for women with a triplet pregnancy: an abandoned randomised controlled trial and meta-analysis. $B M C$ Pregnancy Childbirth 2005;5:8.

24. Hartikainen-Sorri AL, Jouppila P. Is routine hospitalization needed in antenatal care of twin pregnancy. 7 Perinat Med 1984;12:31-4.

25. MacLennan AH, Green RC, O'Shea R, et al. Routine hospital admission in twin pregnancy between 26 and 30 weeks' gestation. Lancet 1990;335:267-9.

26. Mathews DD. A randomized controlled trial of bed rest and sedation or normal activity and non-sedation in the management of non-albuminuric hypertension in late pregnancy. Br 7 Obstet Gynaecol 1977;84:108-14.

27. Leung K, Sum T, Tse C, et al. Is in-patient management of diastolic blood pressure between 90 and $100 \mathrm{~mm} \mathrm{Hg}$ during pregnancy necessary? Hong Kong Med 7 1998;4:211-8.
28. Hobel CJ, Ross MG, Bemis RL, et al. The West Los Angeles Preterm Birth Prevention Project. I. Program impact on high-risk women. Am 7 Obstet Gynecol 1994;170:54-62.

29. Elliott JP, Miller HS, Coleman S, et al. A randomized multicenter study to determine the efficacy of activity restriction for preterm labor management in patients testing negative for fetal fibronectin. 7 Perinatol 2005;25:626-30.

30. Laurin J, Persson PH. The effect of bedrest in hospital on fetal outcome in pregnancies complicated by intra-uterine growth retardation. Acta Obstet Gynecol Scand 1987;66:407-11.

31. Bigelow CA, Factor SH, Miller M, et al. Pilot randomized controlled trial to evaluate the impact of bed rest on maternal and fetal outcomes in women with preterm premature rupture of the membranes. Am 7 Perinatol 2016;33:356-63.

32. Goldenberg RL, Culhane JF, Iams JD, et al. Epidemiology and causes of preterm birth. Lancet 2008;371:75-84.

33. Meher S, Duley L. Rest during pregnancy for preventing pre-eclampsia and its complications in women with normal blood pressure. Cochrane Database Syst Rev 2006;(2):CD005939.

34. Gülmezoglu AM, Hofmeyr GJ. Bed rest in hospital for suspected impaired fetal growth. Cochrane Database Syst Rev 2000;(2):CD000034.

35. Allen C, Glasziou P, Del Mar C. Bed rest: a potentially harmful treatment needing more careful evaluation. Lancet 1999;354:1229-33.

36. Maloni JA, Alexander GR, Schluchter MD, et al. Antepartum bed rest: maternal weight change and infant birth weight. Biol Res Nurs 2004;5:177-86.

37. Stuempfle KJ, Drury DG. The physiological consequences of bed rest. 7 Exerc Physiol Online 2007;10(3):32-41.

38. Hogan MC, Foreman KJ, Naghavi M, et al. Maternal mortality for 181 countries, 1980-2008: a systematic analysis of progress towards Millennium Development Goal 5. Lancet 2010;375:1609-23.

39. Neonatal and perinatal mortality: country, regional and global estimates. Geneva: World Health Organization; 2006. Available: https://apps.who.int/iris/ handle/10665/43444 (accessed 2019 June 21).

40. Global nutrition targets 2025: low birth weight policy brief. Geneva: World Health Organization; 2014.

Affiliations: Program for Pregnancy and Postpartum Health (Matenchuk, Cai, Davenport) and Physical Activity and Diabetes Laboratory (Boulé, Davenport), Faculty of Kinesiology, Sport, and Recreation, University of Alberta; Departments of Medicine (Khurana) and Obstetrics \& Gynecology (Khurana), University of Alberta; Women and Children's Health Research Institute (Cai, Davenport), University of Alberta; John W. Scott Health Sciences Library (Slater), University of Alberta; Alberta Diabetes Institute (Boulé, Davenport), University of Älberta, Edmonton, Alta.

Contributors: Margie Davenport led the review. Rshmi Khurana conceived the project. Margie Davenport, Normand Boulé and Linda Slater designed the search strategy. Margie Davenport, Brittany Matenchuk and Chenxi Cai performed the review. Margie Davenport and Brittany Matenchuk analyzed and interpreted the data. Brittany Matenchuk drafted the manuscript, and Margie Davenport, Rshmi Khurana, Chenxi Cai and Normand Boulé critically revised it for important intellectual content. All of the authors approved the version to be published and agreed to be accountable for all aspects of the work.

Funding: Funding for this project was made possible by the Advancing Women's Heart Health Initiative National and by an Alberta New Investigator Award to Margie Davenport supported by Health Canada and the Heart and Stroke Foundation of Canada. This research was also funded by generous supporters of the Lois Hole Hospital for Women through the Women and Children's Health Research Institute.

Acknowledgement: The authors thank Sandy Campbell from the John W. Scott Health Sciences Library for peer reviewing the database searches.

Supplemental information: For reviewer comments and the original submission of this manuscript, please see www.cmajopen.ca/content/7/3/ E435/suppl/DC1. 